\title{
ON MAPPINGS WITH INTEGRABLE DILATATION
}

\author{
TADEUSZ IWANIEC AND VLADIMIR ŠVERÁK
}

(Communicated by Clifford J. Earle, Jr.)

\begin{abstract}
A factorization of Stoilow's type has been obtained for mappings in $\mathbb{R}^{2}$ with integrable dilatation.
\end{abstract}

\section{INTRODUCTION}

For $\Omega$ a domain in $\mathbb{R}^{n}$ (an open and connected set), we consider a mapping $f: \Omega \rightarrow \mathbb{R}^{n}$ of the Sobolev class $W_{\text {loc }}^{1, n}\left(\Omega, \mathbb{R}^{n}\right)$ with nonnegative Jacobian, $J(x, f) \geq 0$ a.e. We say that $f$ has finite dilatation if

$$
|D f(x)|^{n} \leq K(x) J(x, f) \text { a.e. }
$$

where $1 \leq K(x)<\infty$ for almost every $x \in \Omega$ and $|D f(x)|$ denotes the norm of the differential $D f(x): \mathbb{R}^{n} \rightarrow \mathbb{R}^{n}$.

In recent developments of the nonlinear elasticity theory [Ba, Š], there have been intensive studies of the analytic and geometric properties of such mappings. It is known that the condition $f \in W_{\text {loc }}^{1, n}\left(\Omega, \mathbb{R}^{n}\right)$ does not guarantee that $f$ is continuous, but it does if $f$ has finite dilatation [VG], see also [Ma] for a simpler proof. To state our result we need some definitions.

The dilatation quotient at the points $x \in \Omega$ with $J(x, f) \neq 0$ is defined by

$$
K(x, f)=\frac{|D f(x)|^{n}}{J(x, f)} \geq 1 .
$$

If $J(x, f)=0$, then $D f(x)=0$, and in this case we put $K(x, f)=1$ a.e. Therefore the dilatation function $K(, f): \Omega \rightarrow[1, \infty)$ is defined almost everywhere in $\Omega$. A mapping $f \in W_{\text {loc }}^{1, n}\left(\Omega, \mathbb{R}^{n}\right)$ is said to be $K$-quasi-regular, $1 \leq K<\infty$, if $K(x, f) \leq K$ a.e. If, in addition, $f$ is a homeomorphism, we say that $f$ is $K$-quasi-conformal.

A well-known result in the theory of quasi-regular mappings [Re] states that if $K(, f) \in L^{\infty}(\Omega)$, then $f$ is either constant or an open mapping. In two

Received by the editors September 10, 1991.

1991 Mathematics Subject Classification. Primary 30C62.

Key words and phrases. Quasiconformal mappings, Beltrami equation.

The first author was supported by NSF Grant \#DMS-9007946. The research of the second author was partially carried out during his visit to the University of Bonn, which was supported by SFB 256. 
dimensions this fact has already been recognized by Bojarski [Bo1, Bo2], who has proved Stoilow's type factorization

$$
f=\varphi \circ h^{-1},
$$

with $h: \Omega^{\prime} \rightarrow \Omega$ a homeomorphism (quasi-conformal mapping) and $\varphi: \Omega^{\prime} \rightarrow$ $\mathbb{R}^{2}$ a holomorphic function.

In this note we prove that this factorization remains valid for 2-dimensional mappings whose dilatation function is only assumed to be integrable. Such mappings are, therefore, open and discrete.

Theorem 1. Let $\Omega$ be a bounded domain in the complex plane $(\mathbb{C}, d \sigma(z))$ and, $f \in W^{1,2}(\Omega, \mathbb{C})$ with $J(z, f) \geq 0$ and $K(, f) \in L^{1}(\Omega)$. Then there exists a homeomorphism $h \in W^{1,2}\left(\Omega^{\prime}, \Omega\right)$ and a holomorphic function $\varphi \in$ $W^{1,2}\left(\Omega^{\prime}, \mathbb{C}\right)$ such that

$$
f=\varphi \circ h^{-1} \text {. }
$$

Moreover,

$$
\int_{\Omega^{\prime}}|D h(\omega)|^{2} d \sigma(\omega) \leq \int_{\Omega} K(z, f) d \sigma(z)
$$

and

$$
\int_{\Omega^{\prime}}\left|\varphi^{\prime}(\omega)\right|^{2} d \sigma(\omega) \leq \int_{\Omega}\left|\frac{\partial}{\partial z} f(z)\right|^{2} d \sigma(z) .
$$

One can ask whether some integrability condition on the dilatation function of a mapping $f \in W_{\mathrm{loc}}^{1, n}\left(\Omega, \mathbb{R}^{n}\right)$ with positive Jacobian implies openness also in dimension $n>2$. The arguments we have used in the proof of Theorem 1 suggest the following.

Conjecture 1. Let $\Omega$ be a domain in $\mathbb{R}^{n}$ and $f \in W^{1, n}\left(\Omega, \mathbb{R}^{n}\right)$ with $J(x, f) \geq$ 0 and $K(, f) \in L^{n-1}(\Omega)$. Then $f$ is either constant or an open mapping.

This has already been shown under additional assumptions about the boundary values of $f[\mathrm{Ba}, \check{\mathrm{S}}]$. The general case still remains open. ${ }^{1}$

\section{Preliminaries}

We need a few of the fundamental properties of the quasi-regular mapping in $\mathbb{R}^{n}$. Let us recall the chain rule for differentiation of the composite functions [BI].

Lemma 1.1. Let $f \in W_{\mathrm{loc}}^{1, n}\left(\Omega, \Omega^{\prime}\right)$ be a quasi-regular mapping and let $\varphi \in$ $W_{\mathrm{loc}}^{1, n}\left(\Omega^{\prime}\right)$. Then $\varphi \circ f \in W_{\mathrm{loc}}^{1, n}(\Omega)$, and

$$
D(\varphi \circ f)(x)=(D \varphi)(f(x)) \circ D f(x)
$$

for almost every $x \in \Omega$.

The next result concerns the change of variables in a multiple integral [BI, $\mathrm{Re}, \mathrm{Ri}]$.

\footnotetext{
${ }^{\mathrm{I}}$ Very recently $\mathrm{J}$. Heinonen and $\mathrm{P}$. Koskela confirmed this conjecture for $f$ a "quasi-light mapping" with $K(, f) \in L_{\text {loc }}^{p}(\Omega)$ and $p>n-1$, and most recently for $f \in W_{\text {loc }}^{1, p}\left(\Omega, \mathbb{R}^{n}\right)$ with $p>n$ and $K \in L_{\text {loc }}^{p /(n-1)(p-n)}(\Omega)$.
} 
Lemma 1.2. Let $f: \Omega \rightarrow \Omega^{\prime}$ be quasi-conformal and $u \in L^{1}\left(\Omega^{\prime}\right)$. Then $u(f(x)) J(x, f) \in L^{1}(\Omega)$, and we have

$$
\int_{\Omega^{\prime}} u(y) d y=\int_{\Omega} u(f(x)) J(x, f) d x .
$$

With the aid of these two lemmas we easily arrive at an estimate of the $L^{n}$ norm of the differential of the inverse mapping $h=f^{-1}: \Omega^{\prime} \rightarrow \Omega$ in terms of the dilatation function of $f$.

Lemma 1.3. Let $f: \Omega \rightarrow \Omega^{\prime}$ be a quasi-conformal mapping of bounded domains $\Omega, \Omega^{\prime} \subset \mathbb{R}^{n}$, and let $h: \Omega^{\prime} \rightarrow \Omega$ denote the inverse mapping. Then

$$
\int_{\Omega^{\prime}}|D h(y)|^{n} d y \leq \int_{\Omega} K^{n-1}(x, f) d x .
$$

Proof. We have

$$
\begin{aligned}
\int_{\Omega^{\prime}}|D h|^{n} & =\int_{\Omega}\left|D(f)^{-1}(x)\right|^{n} J(x, f) d x=\int_{\Omega}|\operatorname{adj} D f(x)|^{n} J^{1-n}(x, f) d x \\
& \leq \int_{\Omega}|D f(x)|^{n(n-1)} J^{1-n}(x, f) d x=\int_{\Omega} K^{n-1}(x, f) d x,
\end{aligned}
$$

as desired.

This is why we assumed in Conjecture 1 that $K(, f) \in L^{n-1}(\Omega)$. The last prerequisite deals with the concept and properties of monotone mappings.

We refer to the article of McAuley [McA], in which this subject is well covered by a series of papers.

Let $X$ and $Y$ be compact metric spaces. A continuous mapping $h$ from $X$ onto $Y$ is said to be monotone if for each $y \in Y$ the set $f^{-1}(y)$ is connected. Actually, as shown by Whyburn, this implies that $f^{-1}(C)$ is connected for each connected set $C$ in $Y$.

We shall use the following result of Kuratowski, Lacher, and Whyburn [McA].

Lemma 1.4. If $Y$ is locally connected, then the set of all monotone mappings from $X$ onto $Y$ is closed in $C(X, Y)$. The latter stands for the space of all continuous mappings of $X$ into $Y$ with the topology of uniform convergence.

In our application of this result $X$ and $Y$ will be the 2-spheres, in which case Lemma 1.4 is an elementary exercise.

\section{The Beltrami eQuation}

The space $\mathbb{R}^{2}$ will be identified with the complex plane $\mathbb{C}$, where the area element is denoted by $d \sigma(z)=d x d y, z=x+i y$. For $a \in \mathbb{C}$ and $r>0$, we define the open disk $B(a, r)=\{z ;|z-a|<r\}$ and its boundary $S(a, r)=$ $\{z ;|z-a|=r\}$.

On the extended complex plane $\widehat{\mathbb{C}}=\mathbb{C} \cup\{\infty\}$ we introduce the chordal distance

$$
d(a, \infty)=\frac{2}{\sqrt{1+|a|^{2}}} \text { and } d(a, b)=\frac{2|a-b|}{\sqrt{1+|a|^{2}} \sqrt{1+|b|^{2}}}
$$

if $a, b \neq \infty$. 
Thus $\widehat{\mathbb{C}}$ is a compact metric space. Recall that the chordal distance $d$ is inherited from the Euclidean metric on the 2-sphere via the stereographic projection. It is clear that $d$ restricted to $\mathbb{C}$ induces the same topology as does the Euclidean metric.

We shall make use of the Cauchy-Riemann derivatives. For $f \in W_{\mathrm{loc}}^{1, p}(\Omega, \mathbb{C})$, where $\Omega$ is an open subset of $\mathbb{C}$, the derivatives are defined by

$$
f_{z}=\frac{\partial f}{\partial z}=\frac{1}{2}\left(\frac{\partial f}{\partial x}-i \frac{\partial f}{\partial y}\right), \quad f_{\bar{z}}=\frac{\partial f}{\partial \bar{z}}=\frac{1}{2}\left(\frac{\partial f}{\partial x}+i \frac{\partial f}{\partial y}\right) .
$$

It is straightforward to check the formulas

$$
J(z, f)=\left|f_{z}\right|^{2}-\left|f_{\bar{z}}\right|^{2} \text { and }|D f(z)|=\left|f_{z}\right|+\left|f_{\bar{z}}\right| .
$$

Our proof of Theorem 1 will rest on the existence theorem for the Beltrami equation.

Proposition 2.1 (Bojarski [Bo1, Bo2]). Let $\mu$ be an arbitrary measurable function with compact support and $\|\mu\|_{\infty}<1$. Then, for some $p>2$, there exists a unique solution $f \in W_{\mathrm{loc}}^{1, p}(\mathbb{C}, \mathbb{C})$ of the Beltrami equation

$$
f_{\bar{z}}(z)=\mu(z) f_{z}(z)
$$

such that $f(0)=0$ and $1-f_{z} \in L^{p}(\mathbb{C})$.

This is what we call the normal solution of (2.1) The coefficient $\mu$ is referred to as the complex dilatation of $f$. The normal solution is a quasi-conformal homeomorphism of the extended complex plane, analytic outside the support of $\mu$, and its Taylor expansion at infinity takes the form $f(z)=z+a_{1} z^{-1}+a_{2} z^{-2}=$ $\cdots$. See also [A, L, LV].

For results concerning the existence of solutions of (2.1) with $\|\mu\|_{\infty}=1$, we refer to David [D]; see also [P].

From now on we confine ourselves to those $\mu$ which are supported in the unit disk $\mathbb{B}=\{z ;|z|<1\}$. The purpose of this section is to establish uniform estimates for the inverse mapping $h=f^{-1}: \widehat{\mathbb{C}} \rightarrow \widehat{\mathbb{C}}$. First, recall the inequality

$$
\int_{E}|D h(\omega)|^{2} d \sigma(\omega) \leq \int_{h(E)} K(z, f) d \sigma(z)
$$

for each measurable set $E \subset \mathbb{C}$. Here, the dilatation function of $f$ can be expressed in terms of $\mu$ as

$$
K(z, f)=\frac{|D f(z)|^{2}}{J(z, f)}=\frac{1+|\mu(z)|}{1-|\mu(z)|} .
$$

Proposition 2.2. Let $B_{r}=B(0, r), r>1$, and $\xi, \zeta \in B_{r}$ be such that $|\xi-\zeta|<$ 2. Then

$$
|h(\xi)-h(\zeta)|^{2} \log \frac{4}{|\xi-\zeta|^{2}} \leq \pi \int_{B_{r+3}} K(z, f) d \sigma(z) .
$$

The chordal distance from $h(\xi)$ to $\infty$ is estimated independently of $\mu$ as

$$
d(h(\xi), \infty) \leq \frac{10}{1+|\xi|}
$$

for all $\xi \in \widehat{\mathbb{C}}$. 
Proof. Take notice that $f$ maps $\widehat{\mathbb{C}}-\mathbb{B}$ univalently into $\widehat{\mathbb{C}}-\{0\}$. In view of the Koebe distortion inequality, we can write $|z|+|z|^{-1}-2 \leq|f(z)| \leq|z|+|z|^{-1}+2$, for all $z$ with $|z|>1$; see, for example, [M]. Hence, $B_{r+1} \subset f\left(B_{r+3}\right)$, which is equivalent to

$$
h\left(B_{r+1}\right) \subset B_{r+3} .
$$

We also infer that

$$
|f(z)| \leq 4|z| \text { for }|z| \geq 1 \text {. }
$$

To prove inequality (2.3) we set $|\xi-\zeta|=2 \delta<2$ and $a=\frac{1}{2}(\xi+\zeta) \in B_{r}$. Obviously, $S(a, \delta) \subset B(a, t) \subset B_{r+1}$ if $\delta<t<1$. Since $h$ is a homeomorphism, by the maximum principle, one can find points $\xi^{\prime}, \zeta^{\prime} \in S(a, t)$ such that $|h(\xi)-h(\zeta)| \leq\left|h\left(\xi^{\prime}\right)-h\left(\zeta^{\prime}\right)\right|$. The latter is easily estimated by the integral of $|D h|$ over the circle $S(a, t)$ :

$$
|h(\xi)-h(\zeta)| \leq \frac{1}{2} \int_{S(a, t)}|D h(\omega)||d \omega|
$$

for almost every $t \in(\delta, 1)$. By Hölder's inequality we obtain

$$
t^{-1}|h(\xi)-h(\zeta)|^{2} \leq \frac{\pi}{2} \int_{S(a, t)}|D h(\omega)|^{2}|d \omega| .
$$

Integrating with respect to $t \in(\delta, 1)$, by Fubini's theorem, we find that

$$
\begin{aligned}
-2|h(\xi)-h(\zeta)|^{2} \log \delta & \leq \pi \int_{B(a, 1)}|D h(\omega)|^{2} d \sigma(\omega) \\
& \leq \pi \int_{B_{r+1}}|D h(\omega)|^{2} d \sigma(\omega) .
\end{aligned}
$$

This, together with (2.2) and (2.5), yields

$$
\begin{aligned}
|h(\xi)-h(\zeta)|^{2} \log \frac{4}{|\xi-\zeta|^{2}} & \leq \pi \int_{h\left(B_{r+1}\right)} K(z, f) d \sigma(z) \\
& \leq \pi \int_{B_{r+3}} K(z, f) d \sigma(z),
\end{aligned}
$$

as desired.

Concerning estimate (2.4), it is equivalent to show that $d(z, \infty) \leq$ $10 /(1+|f(z)|)$ for all $z \in \widehat{\mathbb{C}}$. If $|z|>1$ we use (2.6) to obtain

$$
d(z, \infty)=\frac{2}{\sqrt{1+|z|^{2}}} \leq \frac{5 \sqrt{2}}{1+4|z|} \leq \frac{10}{1+|f(z)|} .
$$

For $|z|<1$, in view of the maximum principle $|f(z)| \leq \max _{|\xi|=1}|f(\xi)| \leq 4$, we conclude

$$
\rho(z, \infty) \leq 2 \leq \frac{10}{1+|f(z)|} .
$$

This completes the proof of Proposition 2.2.

\section{Proof of Theorem 1}

We may assume that $\Omega$ is a subdomain of the unit disk, $\Omega \subset \mathbb{B}$, and $f \neq$ constant. Consider the complex dilatation $\mu=\mu(z)$ of $f$; that is,

$$
f_{\bar{z}}=\mu(z) f_{z} \quad \text { a.e. in } \Omega \text {. }
$$


We extend $\mu$ by zero outside $\Omega$ and regard it as a function on the whole of $\mathbb{C}$. For $0<\varepsilon<1$ we define

$$
\mu^{\varepsilon}(z)= \begin{cases}\mu(z) & \text { if }|\mu(z)| \leq 1-\varepsilon, \\ (1-\varepsilon) \mu(z)|\mu(z)|^{-1} & \text { if }|\mu(z)|>1-\varepsilon .\end{cases}
$$

Let $f^{\varepsilon}: \widehat{\mathbb{C}} \rightarrow \widehat{\mathbb{C}}$ be the normal solution of the Beltrami equation

$$
f_{\bar{z}}^{\varepsilon}=\mu^{\varepsilon}(z) f_{z}^{\varepsilon},
$$

and let $h^{\varepsilon}=\left(f^{\varepsilon}\right)^{-1}: \widehat{\mathbb{C}} \rightarrow \widehat{\mathbb{C}}$ denote the inverse mapping. With the aid of Lemma 1.1 we find that

$$
f_{\bar{z}}^{\varepsilon}=-J\left(z, f^{\varepsilon}\right) h_{\bar{\omega}}^{\varepsilon} \quad \text { and } f_{z}^{\varepsilon}=J\left(z, f^{\varepsilon}\right) \overline{h_{\omega}^{\varepsilon}} .
$$

Therefore, (3.3) becomes a quasi-linear equation for $h^{\varepsilon}$ (the hodograph transformation)

$$
h_{\bar{\omega}}^{\varepsilon}=-\mu^{\varepsilon}\left(h^{\varepsilon}(\omega)\right) \overline{h_{\omega}^{\varepsilon}} .
$$

The dilatation function of $f^{\varepsilon}$ can be estimated independently of $\varepsilon$ as

$$
K\left(z, f^{\varepsilon}\right)=\frac{1+\left|\mu^{\varepsilon}(z)\right|}{1-\left|\mu^{\varepsilon}(z)\right|} \leq \frac{1+|\mu(z)|}{1-|\mu(z)|}=K(z, f),
$$

where, in view of our convention, $K(z, f) \equiv 1$ outside $\Omega$. This, combined with Proposition 2.2, leads to uniform estimates

$$
\left|h^{\varepsilon}(\xi)-h^{\varepsilon}(\zeta)\right|^{2} \leq \frac{\pi}{2 \log (2 /|\xi-\zeta|)} \int_{B_{r+3}} K(z, f) d \sigma(z)
$$

for all $\xi, \zeta \in B_{r}, r>1$, with $|\xi-\zeta|<2$, and

$$
d\left(h^{\varepsilon}(\xi), \infty\right) \leq \frac{10}{1+|\xi|} .
$$

A consequence of $(3.5)$ is that the homeomorphisms $h^{\varepsilon}: \widehat{\mathbb{C}} \rightarrow \widehat{\mathbb{C}}$ form an equicontinuous family on each compact subset of $\mathbb{C}$. By the Arzelà-Ascoli theorem it is possible to extract a sequence $h^{\varepsilon_{i}}, i=1,2, \ldots, \varepsilon_{i} \backslash 0$, that converges $c$-uniformly to a mapping $h: \widehat{\mathbb{C}} \rightarrow \widehat{\mathbb{C}}$. Actually, in view of (3.6), the mappings $h^{\varepsilon_{i}}$ converge uniformly on the extended complex plane $\widehat{\mathbb{C}}$ with respect to the chordal metric. According to Lemma 1.4 the limit mapping $h: \widehat{\mathbb{C}} \rightarrow \widehat{\mathbb{C}}$ is monotone. In particular, the set

$$
\mathbf{\Omega}^{\prime}=h^{-1}(\Omega)
$$

is a domain.

Other uniform estimates follow from (2.2) and (2.5), particularly,

$$
\int_{B_{r+1}}\left|D h^{\varepsilon}(\omega)\right|^{2} d \sigma(\omega) \leq \int_{h\left(B_{r+1}\right)} K\left(z, f^{\varepsilon}\right) d \sigma(z) \leq \int_{B_{r+3}} K(z, f) d \sigma(z)
$$

for all $r \geq 1$. This shows that $h^{\varepsilon_{i}}$ converges weakly in $W^{1,2}\left(B_{r+1}\right)$. Thus $h \in W_{\text {loc }}^{1,2}(\mathbb{C})$.

Now we define the function $\varphi: \Omega^{\prime} \rightarrow \mathbb{C}$ by the rule

$$
\varphi(\omega)=f(h(\omega)) \text { for } \omega \in \Omega^{\prime} .
$$


We want to prove that $\varphi$ is holomorphic. To this end, fix an arbitrary open subset $U \subset \Omega^{\prime}$, compactly contained in $\Omega^{\prime}$. Thus $h^{\varepsilon_{i}}(U) \subset \Omega$ for sufficiently small $\varepsilon_{i}$, and we can examine the mappings $\varphi^{\varepsilon}: U \rightarrow \mathbb{C}$ for $\varepsilon \in\left\{\varepsilon_{1}, \varepsilon_{2}, \ldots\right\}$ :

$$
\varphi^{\varepsilon}(\omega)=f\left(h^{\varepsilon}(\omega)\right) \text { for } \omega \in U .
$$

Applying the chain rule (see Lemma 1.1) we find that $\varphi^{\varepsilon} \in W^{1,2}(U)$ and

$$
\frac{\partial \varphi^{\varepsilon}}{\partial \bar{\omega}}=f_{z} h_{\omega}^{\varepsilon}+f_{\bar{z}} \overline{h_{\omega}^{\varepsilon}}, \quad \frac{\partial \varphi^{\varepsilon}}{\partial \omega}=f_{z} h_{\omega}^{\varepsilon}+f_{\bar{z}} \overline{h_{\omega}^{\varepsilon}} .
$$

Then, equations (3.1) and (3.4) imply

$$
\begin{aligned}
& \frac{\partial \varphi^{\varepsilon}}{\partial \bar{\omega}}=\left(\mu(z)-\mu^{\varepsilon}(z)\right) f_{z} \overline{h_{\omega}^{\varepsilon}}, \\
& \frac{\partial \varphi^{\varepsilon}}{\partial \omega}=\left(1-\mu(z) \overline{\mu^{\varepsilon}(z)}\right) f_{z} h_{\omega}^{\varepsilon},
\end{aligned}
$$

where $z=h^{\varepsilon}(\omega)$. It follows from the definition of $\mu^{\varepsilon}(z)$ (see formula (3.2)) that

$$
\begin{gathered}
\left|\mu(z)-\mu^{\varepsilon}(z)\right|^{2} \leq \varepsilon\left(1-\left|\mu^{\varepsilon}(z)\right|^{2}\right), \\
\left|1-\mu(z) \overline{\mu^{\varepsilon}(z)}\right|^{2} \leq 1-\left|\mu^{\varepsilon}(z)\right|^{2} .
\end{gathered}
$$

Notice, too, that $J\left(\omega, h^{\varepsilon}\right)=\left(1-\left|\mu^{\varepsilon}\right|^{2}\right)\left|h_{\omega}^{\varepsilon}\right|^{2}$ (see (3.4)).

Now we use the change of variables according to Lemma 1.2 to obtain

$$
\begin{aligned}
\int_{U}\left|\varphi_{\omega}^{\varepsilon}\right|^{2} & \leq \varepsilon \int_{U} J\left(\omega, h^{\varepsilon}\right)\left|f_{z}\left(h^{\varepsilon}(\omega)\right)\right|^{2} d \sigma(\omega) \\
& =\varepsilon \int_{h^{\varepsilon}(U)}\left|f_{z}(z)\right|^{2} d \sigma(z) \leq \varepsilon \int_{\Omega}\left|f_{z}(z)\right|^{2} d \sigma(z) .
\end{aligned}
$$

In much the same way we obtain the estimate

$$
\int_{U}\left|\varphi_{\omega}^{\varepsilon}\right|^{2} d \sigma(\omega) \leq \int_{\Omega}\left|f_{z}(z)\right|^{2} d \sigma(z) .
$$

These two estimates imply that the sequence $\varphi^{\varepsilon_{i}}=f\left(h^{\varepsilon_{i}}\right)$ converges to the mapping $\varphi=f(h)$ not only pointwise (because $f$ is continuous) but also weakly in $W^{1,2}(U)$. In conclusion, $\varphi \in W^{1,2}(U)$, and we have

$$
\frac{\partial \varphi}{\partial \bar{\omega}}=0, \quad \int_{U}\left|\varphi_{\omega}\right|^{2} d \sigma(\omega) \leq \int_{\Omega}\left|f_{z}\right|^{2} d \sigma(z) .
$$

By the Weyl lemma $\varphi$ is holomorphic in $U$. Since $U$ was an arbitrary compact subdomain of $\Omega^{\prime}, \varphi$ is holomorphic in $\Omega^{\prime}$ as well. This also implies inequality $(0.6)$. To derive inequality $(0.5)$ we use Lemma 1.3

$$
\begin{aligned}
\int_{U}|D h|^{2} d \sigma(\omega) & \leq \lim _{\varepsilon \rightarrow 0} \int_{U}\left|D h^{\varepsilon}\right|^{2} d \sigma(\omega) \leq \lim _{\varepsilon \rightarrow 0} \int_{h^{\varepsilon}(U)} K\left(z, f^{\varepsilon}\right) d \sigma(z) \\
& \leq \int_{\Omega} K(z, f) d \sigma(z) .
\end{aligned}
$$

Of course, $U$ can now be replaced by $\Omega^{\prime}$, proving $(0.5)$.

What remains is to show that $h: \Omega^{\prime} \rightarrow \Omega$ is a homeomorphism, which is the same as to show that $h$ is one-to-one. 
Recall that our function $f \in W^{1,2}(\Omega)$ is actually continuous and nonconstant. For a given point $a \in \Omega$, its preimage $h^{-1}(a) \subset \Omega^{\prime}$ is a continuum (compact connected set) because $h$ is a monotone mapping. Clearly, the analytic function $\varphi=f \circ h$ is constant on $h^{-1}(a)$. Hence $h^{-1}(a)$ consists of a single point, because otherwise $\varphi$ would be constant on the whole of $\Omega^{\prime}$; thus, $f$ would be constant on $\Omega$.

In conclusion, $h: \Omega^{\prime} \rightarrow \Omega$ is a homeomorphism, and we have the factorization $f=\varphi \circ h^{-1}$.

This proves Theorem 1 .

\section{ACKNOWLEDGMENT}

Both authors would like to thank the organizers of the conference in Cortona, May 1991, for hospitality and support. We thank John Ball and Stefan Müller for stimulating discussions.

\section{REFERENCES}

[A] L. V. Ahlfors, Lectures on quasiconformal mappings, Van Nostrand, New York, 1966.

[Ba] J. M. Ball, Global invertability of Sobolev functions and the interpenetration of matter, Proc. Roy. Soc. Edinburgh Sect. A 88 (1981), 315-328.

[Bo1] B. V. Bojarski, Homeomorphic solutions of Beltrami systems, Dokl. Akad. Nauk USSR 102 (1955), 661-664.

[Bo2] _ Generalized solutions of a system of the first order differential equations of elliptic type with discontinuous coefficients, Mat. Sb. 43 (1957), 451-503.

[BI] B. V. Bojarski and T. Iwaniec, Analytical foundations of the theory of quasiconformal mappings in $\mathbb{R}^{n}$, Ann. Acad. Sci. Fenn. Ser. A I Math. 8 (1983), 257-324.

[D] G. David, Solutions de lequation de Beltrami avec $\|\mu\|_{\infty}=1$, Ann. Acad. Sci. Fenn. Ser. A I Math. 13 (1988), 25-70.

[L] O. Lehto, Homeomorphic solutions of a Beltrami differential equation, Festbond 70, Geburstag R. Nevanlinna, Springer, Berlin, 1966, pp. 58-65.

[LV] O. Lehto and K. Virtanen, Quasiconformal mappings in the plane, Springer-Verlag, New York and Heidelberg, 1973.

[Ma] J. Manfredi, Monotone Sobolev functions, preprint, University of Pittsburgh.

[M] A. I. Markushevich, Theory of functions of a complex variable, Prentice-Hall, Englewood Cliffs, NJ, 1967.

[McA] L. F. McAuley, Monotone mappings and open mappings, Proc. of the conference in 1970, State University of New York at Binghamton.

[P] I. N. Pesin, Mappings that are quasiconformal in the mean, Dokl. Akad. Nauk USSR 187 (1969), 740-742; English transl., Soviet Math. (Iz. VUZ) 10 (1969), 939-941.

[Re] Yu. G. Reshetnyak, Space mappings with bounded distortion, Transl. Math. Monographs, vol. 73, Amer. Math. Soc., Providence, RI, 1989.

[Ri] S. Rickman, Quasiregular mappings (to appear).

[Š] V. Šverák, Regularity properties of deformations with finite energy, Arch. Rational Mech. Anal. 100 (1988), 105-127.

[VG] S. K. Vodopyanov and V. M. Goldstein, Quasiconformal mappings and spaces of functions with generalized first derivatives, Siberian Math J. 17 (1977), 515-531.

Syracuse University, Carnegie hall, Syracuse, New York 13244

Heriot-Watt University, Riccarton, Edinburgh EH14 4AS, United Kingdom 